\title{
ORALIDAD MUSICAL Y TECNOLOGÍA \\ Desnaturalizando la partitura como forma de pensamiento
}

\author{
MUSICAL ORALITY AND TECHNOLOGY
}

Denaturing the score as a way of thinking music

\author{
JOAQUÍN BLAS PÉREZ \\ joaquinperez@fba.unlp.edu.ar \\ Laboratorio para el Estudio de la Experiencia Musical. Facultad de Bellas Artes Universidad \\ Nacional de La Plata. Argentina
}

Recibido 3/6/2019 | Aceptado 27/9/2019

\section{Resumen}

Se señala con frecuencia la necesidad de nuevas epistemologías para el abordaje de la música, sobre todo en referencia a las prácticas musicales que no utilizan o utilizan parcialmente la notación. Se propone aquí pensar la música en el marco de una idea ampliada de oralidad como contraparte a la comprensión habitual basada en la escritura. Atender a las prácticas de oralidad musical que trascienden a aquello que podemos objetivar en la partitura nos lleva a ubicar el foco de nuestro análisis en la performance y la interacción social como espacios de construcción de significado. Nos proponemos reflexionar sobre la idea de partitura como tecnología y, posteriormente, de la función de las tecnologías de grabación y de la tecnología digital en tanto configuran diversas formas de oralidad musical.

\section{Palabras clave}

Oralidad; tecnología; música; notación musical; oralidad digital

\section{Abstract}

The need for new epistemologies for the study of music is frequently pointed out, especially in reference to musical practices that do not use or partially use notation. It is proposed here to think about music within the framework of an expanded idea of orality as a counterpart to the habitual understanding based on writing. Attending to musical orality practices that transcend what we can objectify in the score leads us to focus our analysis on performance and social interaction as spaces for the construction of meaning. We intend to reflect on the idea of score as technology, and later on the role of recording technologies and digital technology in that they configure different forms of musical orality.

\section{Keywords}

Orality; technology; music; musical notation; digital orality

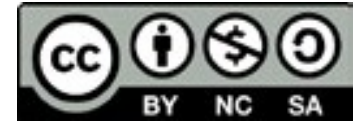


La teoría de la música tal como la conocemos no puede prescindir de la partitura y de la notación musical para explicar sus conceptos. La escritura se convierte, progresivamente a lo largo de la historia, en el espacio por excelencia para objetivar lo musical al punto que algunos músicos, sobre todo en el marco de las ideas del formalismo del siglo XIX, han llegado a contemplar la partitura como si fuera la música misma. Sin embargo, la música preexiste a los sistemas de notación y forma parte de prácticas culturales que se trasmitieron por siglos de manera oral. La etnomusicología reconoce una oralidad musical cantada, tocada, bailada, dramatizada y ritualizada en diferentes culturas (Tokumaru \& Yamaguti, 1986; Aretz, 1993).

Aunque el concepto de oralidad en la lingüística, en la literatura y en la antropología se refiere en principio al habla, la música también forma parte de las prácticas sociales entendidas como orales. En las sociedades primitivas la narración de historias cantadas, la danza y la música no se presentan nunca como manifestaciones autónomas, sino como formas combinadas de memoria étnica y social. En las culturas donde todavía no ha aparecido la escritura, predominan los proverbios y la poesía en acto; el proceso del pensamiento es poético y formulaico por necesidad. De manera similar a lo oral hablado, lo oral musical se vincula fuertemente a lo corporal performativo, que involucra los patrones rítmicos, la respiración y el gesto (Jousse, 1978). La mayoría de las hipótesis antropológicas plantean incluso que la musicalidad de la voz configura una forma expresiva previa al lenguaje hablado (Mithen, 2005); de manera similar la canción como forma performativa musical preexiste a la poesía y la prosa habladas o escritas (Sau, 1972).

El concepto de oralidad ha sido utilizado en los estudios etnomusicológicos de la música medieval (Treitler, 1981) o de las músicas folklóricas oétnicas del Magreb y de Latinoamérica (Salah, 2007; Palominos Mandiola, 2014). Consideramos necesario el concepto de oralidad musical en tanto nos ubica frente a todos los modos de existencia y circulación de la música que no necesariamente están atados a (ni dependen de) lo musical escrito. No nos sorprendemos cuando incluso en la actualidad y en nuestras sociedades hay músicos que son capaces de tocar y cantar aún en el desconocimiento total de la notación musical. En el extremo opuesto estarían los músicos que no pueden tocar o cantar sin la ayuda de una partitura. Nos preguntamos, entonces, qué lugar ocupa lo escrito y qué sucede con todo aquello que queda por fuera.

En primer lugar, resulta clarificador entender, tal como lo hace Walter Ong (1982), la escritura como una tecnología, como una forma artificial de tecnologizar la palabra. Traslademos esta idea a la música, pensemos en la partitura como una tecnología que permitió el registro y la memoria de aquello que se cantaba o tocaba. Esto implica — tanto para la música como para la palabra hablada - desnaturalizar la escritura y recordarnos que lo tocado o cantado puede pensarse, memorizarse y performarse sin necesidad de sus ataduras a lo escrito. Esto resulta a veces difícil de considerar para los músicos y hablantes en sociedades en las que el pensamiento se elabora en sus formas más complejas utilizando los recursos de la escritura. 
En segundo lugar, debemos atender a cómo la escritura, también en el caso de la música, modifica las relaciones sociales y la práctica musical. La partitura, a medida que transcurre la historia, va dejando de ser solo una forma de memoria para convertirse en un espacio de creación. Así, establece nuevas formas de interacción y da lugar a los papeles del compositor, el director o el ejecutante musical. Pero incluso en estas prácticas sobreviven aspectos de la oralidad musical, puesto que el objeto partitura es incapaz de resguardar todos los detalles de la música que deberá ser finalmente performada.

En tercer lugar, debemos considerar lo oral en el marco de la sociedad actual, una sociedad que conoce y en la que circula lo escrito. Resulta útil la idea de oralidad secundaria (Ong, 1982) para definir dicha oralidad, teniendo en cuenta que estaría atravesada por la escritura, pero también por las tecnologías de grabación de audio y las telecomunicaciones del siglo XX y, ya en el XXI, por la tecnología digital e Internet. Es así como nuevas formas de oralidad han sido definidas como oralidad fonográfica, terciaria, digital (Toynbee, 2006; Logan, 2010). La aparición de cada una de estas tecnologías tiene consecuencias similares a la de la aparición de la escritura en el sentido que modifican nuevamente la práctica y las relaciones entre los músicos, lo que reinstala nuevas formas de oralidad que debemos considerar para comprender mejor nuestra disciplina.

Una vez reubicado el papel de la notación musical, cabría preguntarse qué es en realidad todo aquello que circula por fuera, cómo lo analizamos y qué decimos acerca de esa oralidad que discutimos para la música. Si nuestro foco de análisis no se ubica en el lenguaje musical —descripto a partir de conceptos e ideas provenientes de la notación-, ¿cómo hacemos para describir o explicar la música? En principio, el objetivo no radica en abandonar las explicaciones gramaticales en términos de teoría de la música, la idea es incorporar aquello que la teoría de la música descuida. Atender a las prácticas de oralidad nos lleva a ubicar el foco del análisis en los modos performativos a partir de los cuales construimos significado en la interacción social mediada por la tecnología.

\section{La partitura y la ontología de la música como texto}

El tema de la escritura se presenta en la musicología a partir de oponer la idea hegemónica de una ontología de la música como texto a una ontología de la música como performance (Bohlman, 2001; Cook, 1999). La idea de texto va más allá de la partitura como soporte escrito y se encuadra en el marco de la metáfora de la música como lenguaje. En la lingüística la idea de texto se aplica a aquellos enunciados hablados o escritos que están organizados de manera coherente. Puede trasladarse dicha noción al acto musical en tanto consideremos la organización gramatical de la música a partir de ciertas reglas como aquellas descriptas en los manuales tradicionales de armonía y teoría musical o en teorías generativas como la de Fred Lerdahl y Ray Jackendoff (1983). La noción de texto puede aplicarse no solo en referencia a la escritura musical o texto-partitura, sino también a aquello que se manifiesta como musical sonoro. 
La hegemonía de la ontología de la música como texto se perpetúa en la pedagogía del llamado «modelo conservatorio» que ubica a la lectoescritura como práctica central (Musumeci, 2002). Lydia Goehr (1992) critica este modelo y enmarca dichas ideas en el contexto del idealismo romántico y, posteriormente, formalista, donde la obra musical autónoma es considerada autoexpresión del compositor, perfectamente formada y completa. Desde su surgimiento en el siglo IX, los sistemas de notación solían estar incompletos y, de esta manera, permitían la ornamentación y la improvisación; tales aspectos fueron progresivamente eliminados y dieron lugar a la estricta determinación de lo musical por parte del compositor (Born, 2005).

Este modelo, en el que la obra musical como objeto-texto es comunicada de manera lineal en la ejecución, es fuertemente criticado por no considerar la importancia de las dimensiones socioculturales de la práctica que sí serían consideradas en una ontología de la música como performance (Bohlman, 2001; Moran 2014). El concepto de performance musical suele resultar confuso para lectores vinculados a otras áreas disciplinares; diremos por ahora que se lo ha utilizado históricamente como sinónimo de acto o ejecución musical de una partitura, aunque a partir de su revisión en el marco de la musicología crítica y la cognición corporeizada la performance adquiere importantes implicancias en la construcción del significado musical (Martínez, 2017; Johnson, 2007).

Nicholas Cook $(1999,2003)$ propone como alternativa pensar la partitura como un guion similar al guion teatral — más que como un texto que debe ser reproducido. Comprenderla como un guion es «verla como una serie coreográfica de interacciones sociales en tiempo real entre ejecutantes: una serie de actos mutuos de audición y gestos comunes que representan una visión particular de la sociedad humana» (Cook, 2003, p. 3). Cook avanza en la crítica de la relación entre texto y acto entendiendo que se configura, además, un vínculo equívoco entre estructura y performance. Es así como plantea una crítica a las propuestas del funcionalismo cognitivista y del generativismo en música, al afirmar: «Eliminan al sujeto de la práctica y lo reemplazan en la primacía del texto» (Cook, 2003, p. 242). El autor señala que en teorías como la de Lerdahl y Jackendoff (1983) o Eugene Narmour (1990) la dirección seguiría siendo de la estructura a la performance, del texto al acto, por lo que resultarían finalmente tan prescriptivas como la teoría de la musicología tradicional. Dice al respecto que «esta forma de comprender desde la competencia a la performance, del pensamiento abstracto a la realización práctica, constituye el paradigma básico de explicación de todo el pensamiento estructuralista» (Cook, 1999, p. 242). Tomemos como referente a la filósofa posestructuralista y feminista Judith Butler (en Cook, 1999), quien cita las ideas sobre la construcción de la identidad de género en las que afirma que «la identidad se construye performativamente» (p. 243), es decir, invirtiendo la dirección en la construcción del concepto de género ya que de otra manera la estructura resulta de por sí prescriptiva.

En este punto, el lector podría considerar que el modelo de comunicación lineal que conlleva la primacía de la música como objeto o texto trasciende el hecho de que la 
misma haya sido o no escrita en partitura. La consideración de la partitura como guion de Cook resulta interesante puesto que le otorga un papel más flexible para un intérprete que adopte dicha posición. Sin embargo, esta modificación podría, tal como lo sugiere Philip Auslander (2006), no alcanzar para evitar el privilegio del texto en la dicotomía performance/obra a ser interpretada. Por nuestra parte sostendremos que la condición de texto de las músicas que utilizan la notación es diferente de aquellas que se enmarcan en prácticas de oralidad y resulta en cierta manera prescriptiva, sea considerada o no como un texto a ser reproducido.

Si la idea de texto en la música está asociada en primer lugar a su organización gramatical como lenguaje, no debemos dejar de observar las condiciones en las que dichas gramáticas emergen. Es la partitura que en cuanto tecnología permitió el desarrollo de la polifonía y el sistema tonal. La música como lenguaje, en el marco de dicho sistema de organización de la altura, se expande y da lugar a nuevas y complejas gramáticas, es decir, a textos que son el resultado de las sucesivas interacciones de los compositores y ejecutantes con la partitura. Podemos considerar una idea de texto para las prácticas de oralidad, pero ciertamente no nos estaremos refiriendo a la condición de texto-partitura que se le adjudica a la práctica que involucra la composición de una obra y su posterior ejecución. El error de la musicología se produce al otorgar a las prácticas de oralidad musical la misma condición de texto que a la música compuesta y escrita. Pero ¿qué es precisamente lo que las diferencia?

\section{La oralidad y las tecnologías de grabación del sonido}

En las músicas orales no escritas de tradición étnica o folklórica la oralidad se apoya sobre todo en la repetición y la patternización —acumulación de fórmulas breves y recordablescomo modos cognitivos de la memoria y de la performance. En la actualidad, la oralidad musical se encuentra atravesada por todo tipo de tecnologías a partir de las cuales la práctica musical toma distintos modos de existencia y de producción que combinan lo oral y/o lo escrito. La aparición de las tecnologías de grabación del sonido en el siglo XX, por ejemplo, conlleva una nueva forma de registro de la memoria sonora que la define como práctica de oralidad secundaria o fonográfica (Toynbee, 2006). Un ejemplo concreto lo representa la grabación de músicos de blues, góspel y jazz afroamericanos en Estados Unidos a principios del siglo XX. El blues, una música originalmente oral, comienza a ser grabada y vendida; es en este proceso que evoluciona como estilo y como lenguaje. La estructura formal-tonal fija del blues configura una fórmula heredera de la tradición oral y es por esto que en un sentido ontológico puede considerarse el blues como opuesto al ideal de obra de arte romántica. La oralidad fonográfica cambia las restricciones tradicionales de la memoria en el blues y en otras músicas populares. Los discos comienzan a ser parte del material sobre el cual trabajan las nuevas generaciones de músicos y llevan a la evolución de estilos tan diversos como el jazz, el tango o las músicas centroamericanas con gramáticas y formas de textualidad propias. 
Mientras que la ontología de la música como texto-partitura tendería a eliminar lo social como parte de la realización musical, las prácticas que son parte de una oralidad fonográfica la maximizarían. Un nuevo tipo de montaje lateral y procesal entre los agentes involucrados en el hacer la música se contrapone a la ontología de la obra de arte dependiente de un montaje jerárquico y verticalista. La música está embebida en lo social, en su expansión y desarrollo se ha apoyado en la performance y la recepción oral del fonograma. Georgina Born (2005) pone al jazz como ejemplo paradigmático de acto creativo participativo en el que se borra la jerarquía y la separación entre el creador y el performer. La calidad de creatividad retrasmitida en la grabación estaría dada en el marco histórico de la recreación de lo musical grabado en nuevas performances a lo largo de la historia. Esta condición oral ha sido descripta por la etnomusicóloga Ingrid Monson (1996) como parte de un fenómeno de intertextualidad musical o intermusicalidad. Paul Berliner (1994) analiza el fenómeno de intermusicalidad mediante el ejemplo de la recurrencia de un mismo motivo melódico en diferentes grabaciones de músicos en cuarenta y seis años de historia del jazz. Este tipo de ontología de la música difiere de manera considerable de la ontología de la música como texto-partitura y se apoya fundamentalmente en su dimensión performativa, sonora, social y participativa.

\section{Desarrollo tecnológico y oralidad digital}

Durante el siglo XX, el desarrollo de la tecnología de grabación en estudio modifica el proceso de realización musical y gesta nuevas prácticas de oralidad. Entre estas innovaciones se incluyen las reverbs artificiales, la utilización de numerosos micrófonos, el trabajo de mezcla de la grabación en estéreo y, finalmente, la tecnología multipistas. El cambio en las condiciones de producción de fonogramas se produce en paralelo con la aparición del rock and roll y la música pop (Kealy, 1979) e implica la búsqueda y el desarrollo de un sonido. El productor y el ingeniero de grabación son nuevos actores en un proceso de realización musical que tiene como fin la grabación (Théberge, 1989). Las condiciones sociales y la condición ontológica de la música se modifican, el sonido y la grabación se configuran como nuevas formas de existencia de la música. La oralidad fonográfica potencia el carácter colaborativo y democrático al integrar la improvisación y el potencial del estudio de grabación como una ampliación de las formas de interacción grupal en la música (Cuttler, 1984). A pesar de esto, el estudio se establece como un lugar de trabajo de carácter racional y alienizante en un proceso que separa a los individuos y simula la copresencia, pero elimina el mutualismo de la interacción en tiempo real (Théberge, 1989). La música está hecha ahora de fragmentos de performance y de músicas que son ensambladas.

Sin importar la valoración positiva o negativa que hagamos de la condición de fragmentación, estandarización o patternización de la música popular en el marco de la oralidad fonográfica, debemos considerar que en general se las evalúa desde una perspectiva de unidad que proviene de la idea de música como obra de arte. A pesar de esto, la distancia que las prácticas en el estudio de grabación establecen con la 
performance en vivo es real y hace que debamos considerar procesos diferentes para la música en vivo y la grabación en estudio. La aparición de los DJ como consecuencia del desarrollo de los sistemas de audio lleva a la ontología de la música como sonido grabado a un extremo en el que se elimina parte de la performance en vivo. La creación de bases para el rap y el hiphop completa la primera etapa de creación musical a partir de la combinación de fragmentos de músicas ya grabadas, que da lugar a una nueva forma de música improvisada en vivo (Born, 2005). Desde los años setenta podemos ubicar el surgimiento del turntablism como arte de utilizar el mezclador como instrumento en estilos como el house y el techno, lo que le devuelve algo de la dimensión performativa a la práctica del DJ.

En la era digital, la música adquiere una nueva dimensión que trasciende su condición como grabación; la música se convierte en información numérica, en bits y, finalmente, en ceros y en unos (Taylor, 2001). Los dispositivos tecnológicos expanden las condiciones de memoria de lo musical y, al mismo tiempo, los modos de distribución y comunicación. En relación con la oralidad se ha conceptualizado como oralidad terciaria u oralidad digital a la comunicación por Internet desde todo tipo de dispositivos digitales (Logan, 2010). Born (2005) define a este tipo de interacción como un modo de distribución social retransmitida de la agencia creativa. El acceso doméstico a dichas tecnologías y el intercambio de contenidos en Internet modifica profundamente el modo en el que se construye socialmente lo musical al punto que se afirma que la irrupción de lo digital supone «el cambio más fundamental en la historia de la música de Occidente desde la invención en la notación en el siglo IX» (Taylor, 2001, p. 3).

En la producción musical del siglo XXI, sobre todo en aquella vinculada a la música electrónica (EDM o Electronic Dance Music) o en los estilos musicales que toman herramientas de la misma, se plantea una continuidad entre los contenidos musicales que son grabados, retrasmitidos, ensamblados o recreados que resulta similar a aquella que señalamos para el blues. Es la tecnología la que permite el recorte, la edición, la manipulación y el ensamblaje de fragmentos musicales. En estos términos se recupera la cita, la parodia y otros recursos de lo oral. El sample - muestra o fragmento de sonido grabado - se configura como un nuevo material musical. La agencia creativa es distribuida entre diferentes agentes humanos y no humanos. Internet, el mp3, el video digital y las plataformas en las que se comparten estos contenidos moldean en la actualidad nuestro modo de entender la música. Las coincidencias con la intermusicalidad planteada por Monson (1996) para el jazz son numerosas, aunque las condiciones en las que se establezca el intercambio entre interactores sean diametralmente opuestas. Mientras que en el jazz el intercambio se establece en el marco de un mutualismo colaborativo donde la performance grupal configura el espacio de trabajo por excelencia, en la música electrónica y digital la separación entre actores es moneda corriente. A pesar de esto y de manera paradójica, el desarrollo de las tecnologías de transferencia de datos y de mediación tecnológica hace que esta separación se reduzca cada día, que la copresencia virtual sea cada vez más real y la mediación cada vez menos perceptible. 


\section{Consideraciones finales: desnaturalizar la partitura}

Hemos planteado el concepto de oralidad musical como necesario para comprender ciertas formas de práctica musical más allá de la ontología de la música como textopartitura. Desarrollamos, además, la idea de la partitura como tecnología sobre la cual se establecen ciertos tipos de interacción y de vínculos sociales entre los músicos basados en la lectoescritura. Consideramos, también, el desarrollo de ciertas gramáticas o formas de lenguaje musical que se sostienen a partir del uso de la notación musical y la introducción de otros modos de textualidad o gramáticas basadas en diferentes formas de oralidad musical: primaria, fonográfica y digital. En relación con la oposición texto-partitura y performance optamos por considerar que la idea de texto es aplicable también a las formas de oralidad musical pero que se diferencia de la idea de texto-partitura por las formas de interacción, mediación tecnológica y formas de performance que permite. Habría ciertas gramáticas de la oralidad para las cuales deberían desarrollarse nuevas herramientas analíticas y teóricas de lo musical, dichas herramientas no deberían estar basadas o, por lo menos, no solo basadas en aquellas descripciones utilizadas para el texto-partitura. Es por esto que planteamos desnaturalizar la partitura y el lenguaje musical descripto en términos de notación como única forma de pensamiento musical.

La tecnología modifica constantemente el modo en el que nos vinculamos con la música, incluso nuestro modo de entender lo que la música es, es decir, su condición ontológica. Día a día el soporte de datos digitales modifica nuestra experiencia; tanto como antes lo hizo la aparición de las tecnologías de grabación y reproducción de audio o como, a partir del siglo XI, lo hizo el desarrollo del sistema de notación musical. Podemos preguntarnos si en la medida que se desarrolle la tecnología digital el texto-partitura deje alguna vez de ser necesario para el hacer musical. Quizás nuevas formas de teorizar la práctica musical modifiquen aquellas disponibles en la actualidad. Aunque todavía no podemos responder estas preguntas, es necesario recordarnos, como músicos y como docentes, que las ideas musicales desde su concepción hasta su realización ya no dependen exclusivamente de la mediación del soporte escrito. La composición tradicional se convierte en un medio más entre otros. A pesar de esto, la escritura musical y la partitura no pierden vigencia. Tanto en las instituciones de enseñanza como en los contenidos digitales que se comparten por las redes sociales, la partitura sigue teniendo un lugar relevante. En otros casos, el trabajo individual o colectivo sobre materiales que antes fueron producidos y grabados encuentra formas de oralidad que denotan una creatividad retransmitida. Podríamos preguntarnos a futuro, ¿dónde termina la escritura y dónde comienza lo oral en la música? ¿Qué aportes hacen las nuevas tecnologías para una rejerarquización de la oralidad musical en la actualidad? ¿Qué implicancias epistemológicas y pedagógicas conlleva el hecho de que intentemos ser consecuentes y estar a la altura de estos procesos? Se espera que la reflexión sobre las diversas formas de oralidad musical nos permita en un futuro cercano desarrollar nuevas metodologías de estudio, nuevas herramientas de análisis y nuevas pedagogías para la enseñanza musical que trasciendan pero que, a la vez, incluyan de formas creativas a los sistemas de notación musical. 


\section{Referencias}

Aretz, I. (1993). Música y oralidad. Oralidad. Anuario para el rescate de la tradición oral de América Latina y el Caribe, 5, 5-6. Recuperado de http://www.lacult.unesco.org/inmaterial/ oralidad_05_indice.php?uid_ext=\&getipr=\&lg=1

Auslander, P. (2006). Musical Personae. The Drama Review, 50(1), 100-119.

Berliner, P. (1994). Thinking in Jazz: The Infinite Art of Improvisation. Chicago, Estados Unidos: University of Chicago Press.

Bohlman, P. (2001). Ontologies of Music. En N. Cook y M. Everist (Eds.), Rethinking Music (pp. 17-34). Oxford, Inglaterra: Oxford University Press.

Born, G. (2005). On Musical Mediation: Ontology, Technology and Creativity. Twentieth Century Music, 2(1), 7-36. doi:10.1017/S147857220500023X

Cook, N. (1999). Analysing Performance and Performing Analysis. En N. Cook y M. Everist (Eds.) Rethinking Music. (pp. 239-261). Oxford, Reino Unido: Oxford University Press.

Cook, N. (2003). Music as Performance. En M. Clayton, T. Herbert y R. Middleton (Eds), The Cultural Study of Music. A Critical Introduction (pp. 204-214). Londres, Inglaterra: Routledge.

Cutler, C. (1984). Technology, Politics, and Contemporary Music: Necessity and Choice in Musical Forms. Popular Music, 4, 279-300. doi: 10.1017/S0261143000006267

Goehr, L. (1992). The Imaginary Museum of Musical Works: An Essay in the Philosophy of Music. Oxford, Inglaterra: Oxford University Press.

Johnson, M. (2007). The Meaning of the Body. Aesthetics of Human Understanding. Chicago, Estados Unidos: The University of Chicago Press.

Jousse, M. (1978). Le Parlant, la parole, et le souffle. París, Francia: Gallimard.

Kealy, E. R. (1979). From craft to art: the case of sound mixers and popular music. Sociology of Work and Occupations, 6(1), 3-29. doi: 10.1177/009392857961001

Lerdahl, F. y Jackendoff, R. (1983). A Generative Theory of Tonal Music. Cambridge, Estados Unidos: MIT Press.

Logan, R. K. (2010). Understanding New Media: Extending Marshall McLuhan. Nueva York, Estados Unidos: Peter Lang.

Martínez, I. C. (2017). Hacer sentido con el cuerpo en la música. La realidad ampliada de la cognición musical. Revista Argentina de Musicología, (19), 43-58. Recuperado de http://sedici. unlp.edu.ar/handle/10915/77482 
Mithen, S. J. (2005). The singing Neanderthals: The origins of music, language, mind and body. Londres, Inglaterra: Weidenfeld \& Nicholson.

Monson, I. (1996). Saying Something: Jazz Improvisation and Interaction. Chicago, Estados Unidos: University of Chicago Press.

Moran, N. (2014). Social implications arise in embodied music cognition research which can counter musicological «individualism». Frontiers in Psychology, 5, 1-10. doi: 10.3389/ fpsyg.2014.00676

Musumeci, O. (2002). Hacia una educación de conservatorio humanamente compatible. Actas de la Segunda Reunión Anual de SACCOM. Recuperado de http://saccom.org.ar/v2016/node/55

Narmour, E. (1990). The Analysis and Cognition of Basic Melodic Structures: The ImplicationRealization Model. Chicago, Estados Unidos: University of Chicago Press.

Ong, W. J. (1982). Orality and Literacy. The Technologizing of the Word. Londres, Inglaterra: Methuen \& Co Ltd.

Palominos Mandiola, S. (2014). Entre la oralidad y la escritura. La importancia de la música, danza y canto de los Andes coloniales como espacio de significación, poder y mestizajes en contextos de colonialidad. Revista musical chilena, 68(222), 35-57. Recuperado de https:// revistamusicalchilena.uchile.cl/index.php/RMCH/article/view/35910

Salah, F. (2007). Algunos aspectos de la oralidad musical en el Magreb. Revista Oráfrica, 3, 1534. Recuperado de https://www.raco.cat/index.php/Orafrica/article/view/136766/186955

Sau, V. (1972). Historia antropológica de la canción. Barcelona, España: Ediciones Picazo.

Taylor, T. D. (2001). Strange Sounds. Music, Technology and Culture. Londres, Inglaterra: Routledge.

Théberge, P. (1989). The «Sound» of Music: Technological Rationalisation and the Production of Popular Music. New Formations, 8, 99-111.

Tokumaru, Y. y Yamaguti, O. (1986). The Oral and Literate in Music. Tokyo, Japón: Academic Music.

Toynbee, J. (2006). Copyright, the Work and Phonographic Orality. Music Social and Legal Studies, 15(1), 77-99. doi: 10.1177/0964663906060976

Treitler, L. (1981). Oral, Written, and Literate Process in the Transmission of Medieval Music. Speculum, 56(3), 471-491. 